\title{
Wannier-based definition of layer polarizations in perovskite superlattices
}

\author{
Xifan Wu, Oswaldo Diéguez, Karin M. Rabe, and David Vanderbilt \\ Department of Physics and Astronomy, Rutgers University, Piscataway, New Jersey 08854-8019, USA
}

(Dated: June 28, 2018)

\begin{abstract}
In insulators, the method of Marzari and Vanderbilt [Phys. Rev. B 56, 12847 (1997)] can be used to generate maximally localized Wannier functions whose centers are related to the electronic polarization. In the case of layered insulators, this approach can be adapted to provide a natural definition of the local polarization associated with each layer, based on the locations of the nuclear charges and one-dimensional Wannier centers comprising each layer. Here, we use this approach to compute and analyze layer polarizations of ferroelectric perovskite superlattices, including changes in layer polarizations induced by sublattice displacements (i.e., layer-decomposed Born effective charges) and local symmetry breaking at the interfaces. The method provides a powerful tool for analyzing the polarization-related properties of complex layered oxide systems.

PACS numbers: 77.22.-d, 77.22.Ej, 77.80.-e, 77.84.Bw
\end{abstract}

Multicomponent superlattices based on the $\mathrm{ABO}_{3}$ perovskite structure have received much attention recently due to the exciting properties they possess as multifunctional materials (see Ref. 1 and references therein). Experimental studies using modern layer-by-layer epitaxial growth techniques have gone hand in hand with accurate first-principles calculations that have helped to interpret experimental results and to guide the search for superlattice compounds with tailored properties. For example, a compositional perturbation that breaks inversion symmetry was predicted 2] to allow tuning of the dielectric and piezoelectric response, as confirmed later when such superlattices could be grown experimentally $\underline{3}, 4]$. In addition, strained-layer superlattices can show a substantial enhancement of spontaneous polarization; such effects have been observed [4] and analyzed using firstprinciples calculations [5, 6].

One issue that has received much attention theoretically is how to quantify the concept of local polarization. This can be very useful in isolating the contributions of constituent layers to dielectric and piezoelectric properties 2, 3], separating the effects of factors such as epitaxial strain and applied electric fields [5, 6, 7, 8], and understanding the enhancement or suppression of spontaneous polarization [5, 6]. A local description is also essential for characterizing and understanding interface contributions to such properties. Among previously proposed local approaches [8, 9], that of Meyer and Vanderbilt 9] has been one of the most commonly used [5, [6, 7]. Based on a linear approximation involving effective charges and small ionic distortions from a higher-symmetry nonpolar reference structure, this simple model captures the essential physics and provides a semi-quantitative description useful for understanding many aspects of the behavior of multicomponent superlattices. However, as we shall discuss, it is neither exact nor unique.

A first-principles method for identifying local dipoles and computing their dipole moments in an extended system has been proposed in Refs. 10, 11], based on ex- pressing the electric polarization in terms of the centers of charge of Wannier functions (WF) 12, 13] that are maximally localized along the direction of interest [14]. The method was successfully applied to analyze the permittivity of ultra-thin $\mathrm{Si}_{-} \mathrm{SiO}_{2}$ heterostructures [10, 11].

Here, we further develop a closely related method and apply them to ferroelectric perovskite superlattices, where the polarization, particularly that along the growth direction $z$, is of central physical importance. We introduce a WF-based expression for the "layer polarization" along $z$ associated with each charge-neutral AO or $\mathrm{BO}_{2}$ layer in an (001) superlattice built from II-VI $\mathrm{ABO}_{3}$ perovskites such as $\mathrm{BaTiO}_{3}, \mathrm{SrTiO}_{3}$, and $\mathrm{PbTiO}_{3}$. Unlike the approach of Ref. 9, the present one is exact (i.e., the sum of LPs relates exactly to the total supercell polarization) and is entirely free of arbitrary choices in its implementation. We will present examples showing how this approach naturally provides an insightful local description of the polarization behavior of perovskite superlattices, both at zero electric field and under nonzero electrical bias, and in particular yields valuable information about the highly localized atomic and electronic rearrangements at the interfaces.

The modern theory of polarization [12] is routinely used to compute the polarization of a crystal as a sum of ionic and electronic (Berry phase) contributions. In the Wannier representation, this takes the form

$$
\mathbf{P}=\frac{1}{V} \sum_{\tau} Q_{\tau} \mathbf{R}_{\tau}-\frac{2 e}{V} \sum_{m} \overline{\mathbf{r}}_{m}
$$

where $\tau$ and $m$ run over ion cores (of charge $Q_{\tau}$ located at $\mathbf{R}_{\tau}$ ) and Wannier centers (of charge $-2 e$ located at $\left.\overline{\mathbf{r}}_{m}\right)$, respectively, in the unit cell of volume $V$. In the case of a II-VI perovskite superlattice, one may hope to decompose the system into neutral "layers" (that is, AO or $\mathrm{BO}_{2}$ subunits) and define a "layer polarization" (LP)

$$
p_{j}=\frac{1}{S} \sum_{\tau \in j} Q_{\tau} R_{\tau z}-\frac{2 e}{S} \sum_{m \in j} \bar{z}_{m}
$$


in which the sums are restricted to entities belonging to layer $j$. Here $S$ is the basal cell area and we are now focusing only on $z$ components. The LP $p_{j}$ thus defined has units of dipole moment per unit area, and the total polarization, with units of dipole moment per volume, is exactly related to the sum of LPs via $P_{z}=c^{-1} \sum_{j} p_{j}$ where $c=V / S$ is the supercell lattice constant along $z$. For such a decomposition to be meaningful, we need (i) to resolve the arbitrariness associated with the positions of the Wannier centers, and (ii) to be satisfied that the Wannier centers can be assigned to layers without ambiguity. We shall show below by example that (ii) is satisfied for the systems of interest, and thus we next turn our attention to issue (i).

As is well known, the Wannier centers $\overline{\mathbf{r}}_{m}=$ $\left\langle W_{m}|\mathbf{r}| W_{m}\right\rangle$ are not unique because the electronic structure is invariant to unitary rotations among the WFs (corresponding, e.g., to different choices of phases of the Bloch functions in k-space). Marzari and Vanderbilt 13 introduced a method for obtaining a unique set of WFs by choosing the ones that minimize the sum of secondmoment spreads (spatial variances) of the WFs. In a three-dimensional system, this involves finding a best possible compromise between minimal spread in $x, y$, and $z$ directions, and an iterative procedure is needed to find this compromise solution.

Here, we are interested only in polarizations along $z$, and can limit ourselves to minimizing the spread only in that direction 10, 11]. Moreover, we can use a hybrid representation of the electronic ground state that is Bloch-like in $x$ and $y$ and Wannier-like only along $z$ [14]. We start from a conventional band-structure calculation carried out on a mesh of reciprocal points $\mathbf{k}=\left(k_{x}, k_{y}, k_{z}\right)$ and adopt the relabeling $\mathbf{g}=\left(k_{x}, k_{y}\right)$ and $k=k_{z}$. That is, each 2D vector $\mathbf{g}$ labels a string of $J$ k-points running along the $z$ direction with separation $b=2 \pi / J c$. Our task is then to transform the Bloch functions $\left|\psi_{\mathbf{g}, n k}\right\rangle(n=1, \ldots, N)$ into hybrid WFs 14] $\left|W_{\mathbf{g}, m}\right\rangle$ $(m=1, \ldots, N)$ via a $1 \mathrm{D}$ Wannier transform, where $N$ is the number of occupied bands. We will then let $\bar{z}_{m}$ in Eq. (2) be the average of $\bar{z}_{m}(\mathbf{g})=\left\langle W_{\mathbf{g}, m}|z| W_{\mathbf{g}, m}\right\rangle$ over the 2D mesh of $\mathbf{g}$ points. Since the $1 \mathrm{D}$ Wannier transform is done independently at each $\mathbf{g}$, we drop the $\mathbf{g}$ label in the following paragraph.

The case of maximally localized WFs in $1 \mathrm{D}$ was treated explicitly in Sec. IV.C.1 of Ref. 13. There, it was shown that the WFs that minimize the spread functional are identical to the eigenfunctions of the projected position operator $P z P$, where $P=\sum_{n k}\left|\psi_{n k}\right\rangle\left\langle\psi_{n k}\right|$ is the band projection operator. It was also shown how they could be obtained from a parallel-transport based construction using the singular value decomposition (SVD) of the overlap matrices between neighboring k-points, $M_{m n}^{(k)}=\left\langle u_{m k} \mid u_{n, k+b}\right\rangle$, where $u_{m k}$ is the periodic part of the Bloch function $\psi_{m k}$.

The SVD is $M=V \Sigma W^{\dagger}$ where $V$ and $W$ are unitary

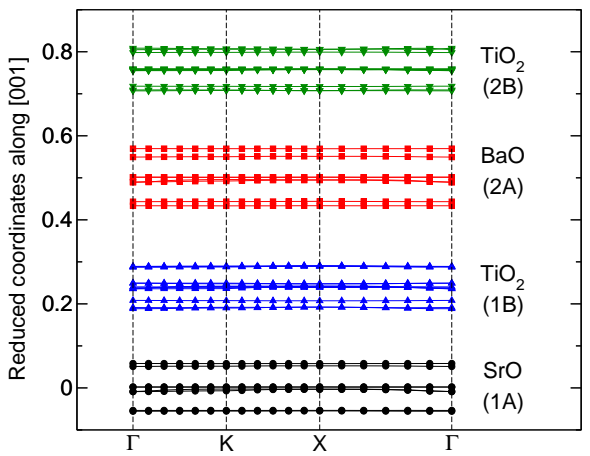

FIG. 1: (Color online.) Dispersion of WF center positions for a $1 \mathrm{BT} / 1 \mathrm{ST}$ superlattice as a function of $\mathbf{g}=\left(k_{x}, k_{y}\right)$.

and $\Sigma$ is positive real diagonal. For small $b, \Sigma$ approaches the unit matrix, and omitting $\Sigma$ to write $\widetilde{M}=U V^{\dagger}$ can be regarded as a way of constructing a purified version of $M$ that is exactly unitary. Then $\Lambda=\prod_{k=1}^{J} \widetilde{M}^{(k)}$ defines a global unitary matrix describing the parallel transport of the states on the k-point string, and its unimodular eigenvalues $\lambda_{m}$ define the Wannier centers via $\bar{z}_{m}=(-c / 2 \pi) \operatorname{Im} \ln \lambda_{m}$. Note that no iterative procedure is required; these 1D Wannier locations can be obtained by a straightforward small-matrix diagonalization. A procedure that is similar in spirit, but slightly different in detail, has recently been proposed elsewhere [15].

In Fig. (1), we present the resulting values of $\bar{z}_{m}(\mathrm{~g})$ for an ab-initio calculation on a 10-atom tetragonal supercell composed of alternating $\mathrm{SrTiO}_{3}(\mathrm{ST})$ and $\mathrm{BaTiO}_{3}$ (BT) units, which we refer to as a $1 \mathrm{ST} / 1 \mathrm{BT}$ superlattice. We did all calculations using the ABINIT code [16], which implements density-functional theory within the local-density approximation (LDA) 17]. We adopted Teter norm-conserving pseudopotentials [18] for which the valence states are $(5 s 5 p 6 s)$ for $\mathrm{Ba},(4 s 4 p 5 s)$ for $\mathrm{Sr}$, $(3 s 3 p 3 d 4 s)$ for $\mathrm{Ti}$, and $(2 s 2 p)$ for $\mathrm{O}$. We used a planewave energy cutoff of $45 \mathrm{Ha}$, a $6 \times 6 \times 3$ Monkhorst-Pack self-consistency mesh, and a $12 \times 12 \times 3$ bandstructure mesh. We assumed perfect epitaxial growth of the superlattices on a cubic ST substrate having a theoretical equilibrium lattice constant of $7.265 \mathrm{bohr}$ and tetragonal P4mm symmetry.

The key feature visible in the $\bar{z}(\mathbf{g})$ dispersion relation in Fig. 1 is that the WF centers separate quite naturally into distinct layers as anticipated. The 1D Wannier positions $\bar{z}$ are almost independent of $\mathbf{g}=\left(k_{x}, k_{y}\right)$, and there are robust gaps between layers. Moreover, we find eight Wannier centers in each $\mathrm{BaO}$ or $\mathrm{SrO}$ layer and 12 in each $\mathrm{TiO}_{2}$ layer (four for each cation semicore shell and four for each oxygen $2 s 2 p$ shell), so that the layers are neutral as expected. All this demonstrates that the proposed Wannier-based approach does indeed lead to a natural and robust decomposition into easily identified neutral layers. 


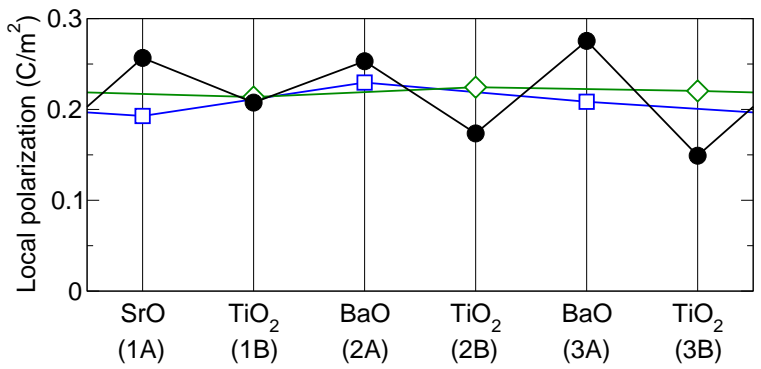

FIG. 2: (Color online.) Local polarization profile of 1ST/2BT supercell, from effective charge approximation based on $\mathrm{A}$ centered (open square) or B centered (open diamonds) analysis, and from layer polarization analysis (filled circles). The overall supercell polarization is $0.22 \mathrm{C} / \mathrm{m}^{2}$.

It is then straightforward to define the LP $p_{j}$ associated with each layer according to Eq. (2). For comparison with other definitions, we also introduce the corresponding local polarization $P_{j}=p_{j} / c_{j}$ having the correct units of polarization (dipole per unit volume), where $c_{j}$ is chosen as half the distance between the two neighboring cations. In Fig. 2] we show the local polarization profile calculated in this way for the case of a $1 \mathrm{ST} / 2 \mathrm{BT}$ superlattice (15-atom supercell). The details are the same as for the calculation of the 1ST/1BT superlattice, except that we use a $6 \times 6 \times 2 \mathrm{k}$-point sampling. We compare our results with the ones obtained from the commonly applied approximate scheme [9] in which the local polarization is estimated by multiplying the Born effective charges of the atoms in a unit cell layer by their displacements relative to a reference structure. The effective charges are obtained from linear response calculations in the ferroelectric ground state. By its very nature, this approximate scheme [9] has only half the spatial resolution of our new scheme, since it applies only to entire $\mathrm{ABO}_{3}$ cells. (Note that it cannot easily be extended down to the resolution of $\mathrm{AO}$ and $\mathrm{BO}_{2}$ layers because the sum of $Z^{*}$ values in such a layer does not vanish, so that the definition would depend on choice of reference structure in an unsatisfactory way.)

The results shown in Fig. 2 are consistent with the findings of previous theoretical studies [5] showing that the $\mathrm{SrTiO}_{3}$ portion of the supercell becomes polarized to almost the same degree as the $\mathrm{BaTiO}_{3}$ portion. However, the improved resolution associated with the new approach is also clearly evident in the figure. For example, one can now see that the polarization tends to be larger in the $\mathrm{AO}$ layers than in the $\mathrm{TiO}_{2}$ layers (see next paragraph). Moreover, our new approach is free of three limitations of the approximate one [5, 6, 7, 9]. First, we avoid the choice between an A- or B-centered analysis. Second, we do not have the problem of choosing an arbitrary local reference structure as the basis for the definition of the atomic displacements. Third, our LPs $p_{j}$ sum to give the exact total polarization of the entire supercell, whereas the approximate ones do not.

The Born effective charges $Z^{*}$, defined as the first derivatives of polarization with respect to atomic displacements, describe the dynamics of the charge transfer induced by such displacements. We now illustrate how the LP concept can be used to decompose the $Z^{*}$ for an atom in one layer into contributions from neighboring layers [19]. This is demonstrated in Table \for the case of a supercell of tetragonal bulk BT that has been tripled along [001] (3BT supercell). Each of the four symmetryinequivalent atoms $\left(\mathrm{Ti}\right.$ and $\mathrm{O}_{\|}$in a $\mathrm{TiO}_{2}$ layer and $\mathrm{Ba}$ and $\mathrm{O}_{\perp}$ in a $\mathrm{BaO}$ layer) was displaced along $z$ in turn, and the changes in all six LPs in the supercell were computed. For $\mathrm{Ba}, \mathrm{O}_{\perp}$ and $\mathrm{O}_{\|}$, the induced polarizations are dominated by contributions from the same atomic layer, at the level of around $45 \%$. In contrast, for the $\mathrm{Ti}$ atom, the contributions from the first neighboring layers are almost as large as from the layer itself. This is consistent with the well-known role of the $\mathrm{Ti}(3 d)-\mathrm{O}(2 p)$ hybridization in giving rise to the anomalous Born effective charges in these perovskites 20], and the fact that the WFs that embody this hybridization reside on the $\mathrm{O}$ atoms. Thus, a motion of the Ti atoms along [001] modulates this hybridization and shifts the centers of the WFs residing on the neighboring $\mathrm{BaO}$ layers. This effect also helps explain why the LPs for AO layers are larger than for $\mathrm{TiO}_{2}$ layers in Fig. 2]

We further illustrate the utility of the LP analysis by considering its behavior in a macroscopic electric field $\mathcal{E}$ 21] applied along [001]. We used a constrainedpolarization mapping technique 22] generalized to include volume relaxation 23. to find the minimum-energy configuration for each given polarization. The resulting LPs $p_{j}$ vs. total polarization are shown for the $1 \mathrm{ST} / 2 \mathrm{BT}$ supercell in the left-hand panels of Fig. 3 We see that each LP is roughly linear in the total polarization for all six layers, but with nonlinearities appearing at large values of polarization. For each data point, we extracted the corresponding macroscopic electric field value, and plotted the LPs against this field in the right-hand panels of Fig. 3 The results show a strongly nonlinear depen-

TABLE I: Layer decomposition of the [001] Born effective charges in a $3 \mathrm{BT}$ supercell. Total effective charges are given in the last row.

\begin{tabular}{lrrrr}
\hline \hline & $\mathrm{Ti}(1 \mathrm{~B})$ & $\mathrm{Ba}(1 \mathrm{~A})$ & $\mathrm{O}_{\|}(1 \mathrm{~A})$ & $\mathrm{O}_{\perp}(1 \mathrm{~B})$ \\
\hline $\mathrm{BaO}(1 \mathrm{~A})$ & 1.433 & 1.268 & -2.448 & -0.225 \\
$\mathrm{TiO}_{2}(1 \mathrm{~B})$ & 1.872 & 0.148 & -0.231 & -0.930 \\
$\mathrm{BaO}(2 \mathrm{~A})$ & 1.262 & 0.434 & -1.027 & -0.191 \\
$\mathrm{TiO}_{2}(2 \mathrm{~B})$ & 0.619 & 0.296 & -0.542 & -0.216 \\
$\mathrm{BaO}_{(3 \mathrm{~A})}$ & 1.211 & 0.435 & -1.046 & -0.348 \\
$\mathrm{TiO}_{2}(3 \mathrm{~B})$ & 0.636 & 0.191 & -0.264 & -0.217 \\
\hline$Z^{*}$ & 7.033 & 2.772 & -5.557 & -2.127 \\
\hline \hline
\end{tabular}




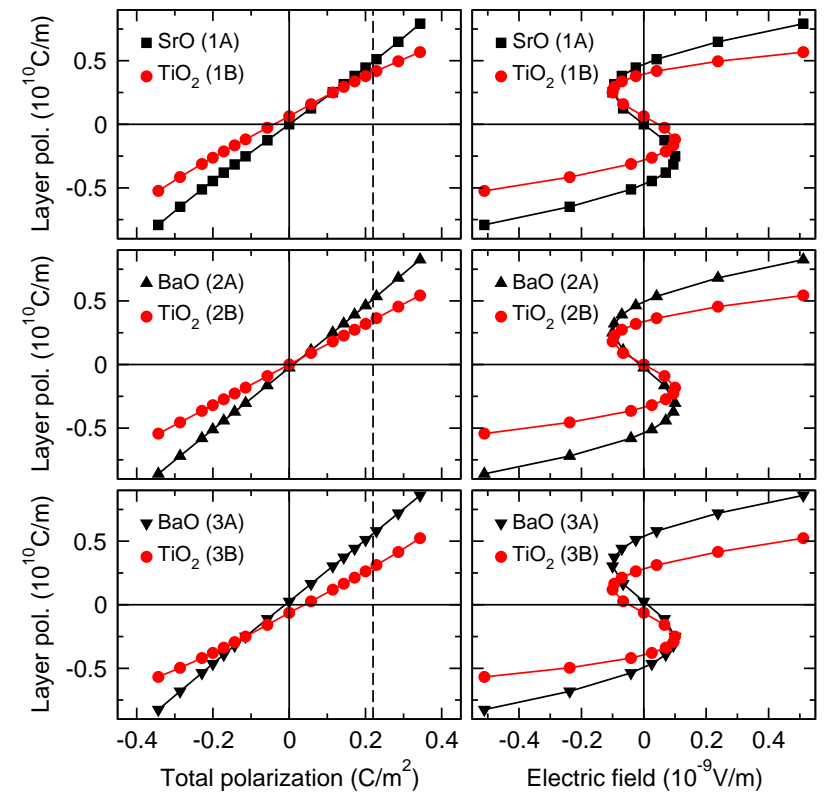

FIG. 3: (Color online.) Layer polarization as a function of (a) total polarization of the supercell, and (b) macroscopic electric field in the supercell, for six consecutive layers in the 1ST/2BT supercell. Labeling of layers follows Fig. 1.

dence, typical of that found for total polarization as a function of electric field in ferroelectric materials.

The most striking features seen in Fig. B are for the two interface layers $\left(\mathrm{TiO}_{2}\right.$ layers $1 \mathrm{~B}$ and $\left.3 \mathrm{~B}\right)$. They show local breaking of inversion symmetry; that is, the LP as a function of macroscopic field does not pass through the origin, at which for the system as a whole the symmetry of the $P 4 / \mathrm{mmm}$ space group requires $\mathbf{P}=0$ and $\mathcal{E}=0$. One of these interface $\mathrm{TiO}_{2}$ layers has a nearest-neighbor $\mathrm{SrO}$ layer above and a $\mathrm{BaO}$ layer below, and the other vice versa, the two interface layers being related by a mirror symmetry. The LP approach give us much more precise information about the response of these interfaces to applied fields than could be obtained from an analysis of either the total polarization, or of the local polarizations as previously defined (involving a smearing over three sequential atomic layers). We expect that this method of analysis of interface layers will be invaluable for identifying the interface contributions to the properties of superlattices with more than two components, particularly those with globally broken inversion symmetry [2, 23]. Similar considerations apply to $\mathrm{BaO}$ layers $2 \mathrm{~A}$ and $3 \mathrm{~A}$, which also see an environment of broken inversion symmetry. For these, however, the symmetry breaking enters only at the level of second-neighbor layers, so the effects are smaller in magnitude.

In summary, we have introduced a definition of the layer polarization (LP) in a multicomponent perovskite superlattice that is exactly related to the polarization of the full system and does not require choosing an arbitrary reference configuration. For each atomic layer, the LP is uniquely determined by the spatial locations of ionic and WF centers, and it can easily be computed in any firstprinciples code as a post-processing step after standard electronic structure calculations. Although this polarization is not directly measurable experimentally, we show examples in which the LP precisely quantifies polar distortions throughout the superlattice, the high resolution of the definition being particularly relevant for inspecting the behavior of interface layers. Immediate applications include modeling of interface effects on total polarization of multicomponent superlattices [23], systematic studies of self-poling effects in superlattices [23], and studies of the coupling of phonons to the interfaces. For superlattices containing magnetic constituents, the spin degeneracy assumption can be relaxed, so that the WF centers will have additional local spin ordering information.

This work was supported by ONR Grant N00014-051-0054 and by the Center for Piezoelectrics by Design under ONR Grant N00014-01-1-0365.

[1] Ph. Ghosez and J. Junquera, in Handbook of Theoretical and Computational Nanotechnology, edited by M. Rieth and W. Schommers, American Scientific (2006).

[2] N. Sai, B. Meyer, and D. Vanderbilt, Phys. Rev. Lett. 84, 5636 (2000).

[3] M. P. Warusawithana, E. V. Colla, J. N. Eckstein, and M. B. Weissman, Phys. Rev. Lett. 90, 036802 (2003).

[4] H. N. Lee, H. M. Christen, M. F. Chisholm, C. M. Rouleau, and D. H. Lowndes, Nature (London) 433, 395 (2005).

[5] J. B. Neaton and K. M. Rabe, Appl. Phys. Lett. 82, 1586 (2003).

[6] S. M. Nakhmanson, K. M. Rabe, and D. Vanderbilt, Appl. Phys. Lett. 87, 102906 (2005).

[7] K. Johnston, X. Huang, J. B. Neaton, and K. M. Rabe, Phys. Rev. B 71, R100103(2005);

[8] M. Sepliarsky, M. G. Stachiotti, and R. L. Migoni, Phys. Rev. Lett. 96, 137603 (2006).

[9] B. Meyer and D. Vanderbilt, Phys. Rev. B 65, 104111 (2002).

[10] F. Giustino, P. Umari, and A. Pasquarello, Phys. Rev. Lett. 91, 267601 (2003).

[11] F. Giustino and A. Pasquarello, Phys. Rev. B 71, 144104 (2005).

[12] R. D. King-Smith and D. Vanderbilt, Phys. Rev. B 47, R1651 (1993); R. Resta, Rev. Mod. Phys. 66, 899 (1994).

[13] N. Marzari and D. Vanderbilt, Phys. Rev. B 56, 12847 (1997).

[14] C. Sgiarovello, M. Peressi, and R. Resta, Phys. Rev. B 64, 115202 (2001).

[15] J. Bhattacharjee and U.V. Waghmare, Phys. Rev. 71, 045106 (2005).

[16] X. Gonze et al., Comput. Mater. Sci. 25, 478 (2002).

[17] P. Hohenberg and W. Kohn, Phys. Rev. 136, B864 (1964); W. Kohn and L. J. Sham, ibid. 140, A1133 (1965).

[18] M. Teter, Phys. Rev. B 48, 5031 (1993). 
[19] See also Sec. VII of Ref. 13 for a discussion of atom-byatom decomposition of the $Z^{*}$ in GaAs.

[20] R. E. Cohen and H. Krakauer, Phys. Rev. B 42, 6416 (1990); Ph. Ghosez, J.-P. Michenaud, and X. Gonze, ibid 58, 6224 (1998).

[21] I. Souza, J. Íñiguez, and D. Vanderbilt, Phys. Rev. Lett.
89, $117602(2002)$.

[22] O. Diéguez and D. Vanderbilt, Phys. Rev. Lett. 96, 056401 (2006).

[23] X. Wu, O. Diéguez, K. M. Rabe, and D. Vanderbilt, in preparation. 